aetiology that causes vaginal discharge but due of the complications that a recurrent BV infection can cause in women especially pregnant women, its management must be effective in gynecologic and obstetric services and also in the campaigns against STIs and HIV that are regularly conducted at the national level.

\section{P3.94 DOES MASS DRUG ADMINISTRATION WITH AZITHROMYCIN FOR TRACHOMA CONTROL HAVE AN IMPACT ON THE PREVALENCE AND MACROLIDE RESISTANCE OF GENITAL MYCOPLASMA GENITALIUM INFECTION?}

M Harrison, ${ }^{1} \mathrm{M}$ Marks, ${ }^{2} E$ Harding-Esch, ${ }^{3,1} \mathrm{MJ}$ Pond, ${ }^{1} \mathrm{R}$ Butcher, ${ }^{2} \mathrm{~A}$ Solomon, ${ }^{2} \mathrm{NK}$ Tan, ${ }^{4} \mathrm{~A}$ Nori, ${ }^{1} \mathrm{H}$ Kako, ${ }^{5} \mathrm{D}$ Mabey, ${ }^{2} \mathrm{ST}$ Sadiq, ${ }^{1,2,4}$. ' Applied Diagnostic Research and Evaluation Unit, Institute of Infection and Immunity, St George's, University of London, UK; ${ }^{2}$ London School of Hygiene and Tropical Medicine, UK; ${ }^{3}$ HIVISTI Department, Public Health UK; ${ }^{4}$ St George's University Hospitals NHS Foundation Trust; ${ }^{5}$ Ministry of Health and Medical Services, Solomon Islands

\subsection{6/sextrans-2017-053264.329}

Introduction The first round of Mass Drug Administration (MDA) with $1 \mathrm{~g}$ oral azithromycin for ocular Chlamydia trachomatis (CT) infection, which is a key component of trachoma control strategies, concomitantly reduced genital CT infection in the Solomon Islands. However, this dose is known to be sub-optimal for the treatment of genital Mycoplasma genitalium (MG) infection and may also encourage emergence of antimicrobial resistance (AMR) to macrolides in MG.

Methods Pre-MDA and 6 months post-MDA CT-negative selfcollected vulvo-vaginal swabs from women attending three outpatient antenatal clinics (Honiara, Solomon Islands), already investigated for the impact of MDA on genital CT prevalence, were tested for MG infection using nucleic acid amplification. MG positive samples were subsequently tested for macrolide resistance by sequencing domain $\mathrm{V}$ of $23 \mathrm{~S}$ rRNA DNA region of MG.

Results MG positivity was found in $11.9 \%$ (28/236) of women pre-MDA and in $10.9 \% \quad(28 / 256) 6$ months post-MDA $(p=0.7467) .22 \mathrm{MG}$ positives from each of the pre-MDA and post-MDA samples were sequenced, all showing a macrolide susceptible genotype.

Conclusion A single MDA round in an island population with apparent high MG prevalence with $1 \mathrm{~g}$ azithromycin did not impact on either MG positivity or detection of genetically determined macrolide resistance in this population, in contrast to decreased genital CT positivity in the same population. It is unclear if this apparent lack of impact is due to inadequate efficacy of single-dose azithromycin or transmission dynamics of the infection. Further investigation of the impact of multiple rounds of MDA on antibiotic-experienced and -naïve populations is warranted.

\section{P3.95 QUALITY OF CARE AND INCIDENCE OF STIS IN A COHORT OF TRANSGENDER WOMEN LIVING WITH HIV}

HEL Reno, R Presti, T Brown, BP Stoner. Division of Infectious Disease, Washington University in St. Louis, MO, USA

10.1136/sextrans-2017-053264.330

Introduction Transgender women are at high risk for HIV infection, but less is understood about their rates of sexually transmitted infections (STIs) and the quality of sexual health services they receive. The Washington University Virology Clinic (St. Louis, MO USA) serves patients living with HIV; $2 \%$ of patients report being transgender women.

Methods We conducted a retrospective cohort study of transgender women living with HIV $(n=41)$ to document frequency of testing for incident bacterial STIs [syphilis, gonorrhoea (GC), chlamydia (Ct)], with demographic information, markers of HIV care, and STI test results from 20112015.

Results Most patients were African American (91\%) and on antiretroviral medications (>88\%), although only $56 \%$ maintained HIV viral load suppression. Health challenges included a history of disrupted antiretroviral treatment $(66 \%)$ and STIs $(65 \%)$. Incident syphilis was diagnosed in $7.3 \%$ patients, and GC and Ct were diagnosed in $19.5 \%$ and $9.8 \%$ of patients, respectively. For syphilis screening, $90 \%$ of patients were tested at least once a year, and $53 \%$ of patients were tested more than once a year. For GC/Ct screening, $89 \%$ of patients were tested at least once a year and $49.3 \%$ of patients were tested more than once a year. For patients with GC or $\mathrm{Ct}$ infection, only $44 \%$ of patients were retested at the site of infection 3-6 months after treatment. Reinfection with GC or $\mathrm{Ct}$ was subsequently diagnosed in $19.5 \%$ of patients. Frequency of three site testing for GC/Ct (genital, rectal, pharyngeal) increased over the course of the study period (from $3 \%$ of patients to $34 \%$ of patients, $\left.\mathrm{X}^{2}=17.69, \mathrm{p}=0.001\right)$.

Conclusion Transgender women living with HIV are at high risk for incident bacterial STIs. Frequency of testing for STIs increased over a five-year period, but many patients with documented infection were not re-tested after treatment as recommended by current guidelines. Understanding STI rates, primary locations of infection, and lack of retesting in patients will improve patient education and standardise care for patients.

\section{P3.96 FACTORS ASSOCIATED WITH COMMERCIAL SEXUAL BEHAVIOUR AMONG MEN WHO HAVE SEX WITH MEN IN SHENZHEN, 2011 TO 2015}

${ }^{1}$ Hong Fuchang, ${ }^{1}$ Cai Yumao, 'Lai Yonghui, 'Wen Lizhang, ${ }^{1} Y u$ Weiye, ${ }^{2}$ Liu Hui. ${ }^{1}$ Shenzhen Centre for Chronic Disease Control and Prevention, Shenzhen, China; ${ }^{2}$ National Centre for AIDS/STD Control and Prevention, China CDC

\subsection{6/sextrans-2017-053264.331}

Introduction With the use of new-type drugs, Money boys (MB) will have unprotected sex and injection behaviours easily, thus accelerate the spread of HIV among men who have sex with men(MSM) through sexual transmission and blood transmission. Our study is to investigate the status and factors associated with commercial sexual behaviour among MSM in Shenzhen, China.

Methods Convenience sampling method was used to recruit MSM from 2011 to 2015. Questionnaire-based interviews were conducted on a one-on-one basis. Data were collected including socio-demographic information, HIV testing history, history of blood donation and drug abuse in recent two years, self-identified sexual orientation, role in homosexual behaviour, ever being $\mathrm{MB}$ and clients of MB. $5 \mathrm{ml}$ blood samples were taken and tested for treponema pallidum and HIV antibodies.

Results Among the total of 3040 MSM recruited,341 (11.2\%) reported ever being MB. The prevalence of syphilis, HIV, and 